\title{
Bouba-Kiki in the plate: combining crossmodal correspondences to change flavour experience
}

\author{
Merle T Fairhurst $^{1 *}$, Deiniol Pritchard ${ }^{2}$, Daniel Ospina ${ }^{2}$ and Ophelia Deroy ${ }^{1}$
}

\begin{abstract}
Background: While recent studies demonstrate the unexpected influence of plate shape and abstract names on the taste and flavour of foods, it is still challenging to derive more specific rules about how to use verbal and visual presentation to affect the experience of a complex dish.

Findings: In the present study, we paired with the Experimental Kitchen at The Fat Duck to manipulate both plate shape and serving presentation of a complex dish and to investigate the relative contributions and interaction of these two factors on the naming and sensory evaluation of the food. The results confirm that certain visual presentations of food, but not plate shape, were associated with unfamiliar names if they respected sound symbolic associations. By contrast, the change in the perceived sweetness of the dishes was driven by the interaction between the shape of the plate and the presentation of the dish.

Conclusions: The study therefore allows us to disentangle the effects of plate shape and dish presentation on the diner's expectations and experience and shows how fundamental research on crossmodal effects can inform the creative process in the kitchen.
\end{abstract}

Keywords: Crossmodal correspondences, Malomu, Kichiki

\section{Findings}

Recent experiments have refuted the idea that vision and taste represent two totally separate sensory domains; not only do pictures of food activate the right insula/operculum and the left orbitofrontal cortex, both gustatory-processing areas [1], but abstract shapes and colours can also evoke basic tastes or complex flavours (see [2] for a recent review): rounded shapes are matched with sweeter tastes or drinks rather than with bitter, salty or sour ones (for example, [3,4]). Moreover, they are more often paired with fruity aromas and soft textures [5-7] in a way which seems consistent across cultures $[8,9]$.

Interestingly, these correspondences between shapes and tastes or flavours appear to fit with the welldocumented correspondence which links shapes and speech sounds. Meaningless words like 'malomu' $[10,11]$ or 'bouba' [12] are also matched with more rounded

\footnotetext{
* Correspondence: merle.fairhurst@sas.ac.uk

${ }^{1}$ Centre for the Study of the Senses, School of Advanced Study, University of London, London WC1E 7HU, UK

Full list of author information is available at the end of the article
}

shapes, as sweet foods do, and these words turn out to be paired, in Western populations at least, with sweeter creamy foods like milk chocolate. Meanwhile, words such as 'kichiki' or 'kiki' which are paired with angular shapes are matched with bitter-tasting foods such as dark chocolate $[7,8]$.

A key challenge, for cognitive neuroscientists and chefs alike, is to know whether these correspondences can impact significantly on a diner's appreciation and perception of a complex dish $[13,14]$. This question has mostly been addressed by looking at how changing the shape of the container (that is, the package or the plate) could affect the experience of a food, whose shape was fixed. In the recent study conducted by [15], participants tasted a rounded piece of cheesecake off of a black or white plate that could be either round or square. Participants rated the dessert as significantly sweeter (by 20\%) and more intense (by 30\%) when tasted off the round white plate than from any of the other three plates. However, this influence could be due in part to a change in the visual appearance of the food on a white 
background, and in part to the familiarity with rounded plates, and not to a crossmodal correspondence between roundedness and sweetness. In another study by Piqueras-Fiszman et al. [16], participants ate a strawberry mousse from a white plate that was either round, square or triangular. In this study, the shape of the plate did not exert any effect on the perceived sweetness of the mousse. A potential confound came from the fact that the food itself had been made into a very angular form: the angularity of the food itself (which was constant across the three plates) may have been foregrounded against the background of the plate's shape in the participant's mind (see [17] for a review).

\section{Methods}

In the present study, we decided to manipulate both the shape of the plate and of the food displayed on it, to see whether these two elements would interact or whether the shape of the food would always be in the foreground and drive the effects on flavour perception. Two groups were tested in the experiment which was conducted during a scientific public engagement event at the Dana Centre in London. Sixteen participants were retained in each group after outliers were excluded on the basis of missing data, age (range: 18 to 50 ) and familiarity with restaurant dining. In each session, participants were served two white plates of the same shape (group 1: 16-cm square plates; group 2: $16-\mathrm{cm}$ diameter round plates). Servings consisted of the same ingredients in the same quantities (three pieces of beetroot, all of the same weight, served with goat curd, micro chard and micro watercress and fried shallots) but presented in different ways. In the rounded presentation, the three pieces of beetroot were cut in half-spheres and displayed in a circle on the surface of the plate, while in the angular presentation, the three pieces of beetroot were cut into pyramids and displayed so as to form a triangle (Figure 1).

Prior to testing, written informed consent was obtained for each subject with ethics approval obtained from the School of Advanced Study, Research Ethics Committee. Research methods were in compliance with the Declaration of Helsinki. The participants in both groups were first presented with a picture of the two presentations (without the context of plate shape). They then received two successive plates of the same shape, with the two presentation types resulting in a congruent and incongruent combination of plate and food: group 1 received a square plate with an angular presentation (congruent) and a square plate with round presentation (incongruent); group 2 received a round plate with a round presentation (congruent) and a round plate with an angular presentation (incongruent). Based on sight alone, for each serving, participants were asked to indicate the best name for the dish. Ratings were performed using an unstructured, visual analogue scale anchored with the words 'malomu' and 'kichiki' - two names chosen from the literature on sound symbolism for being associated, respectively, with rounded and angular visual shapes [18]. Participants were asked to place a mark on the scale between these two names, indicating which best fitted their visual experience of the food presented. These ratings were then converted to a numeral rating ( 1 to 7$)$. After eating the dish, participants were asked to rate the flavor experience in terms of sweetness, sourness, pleasantness and intensity with a numerical rating ranging from 0 to 10 , where 0 was not at all and 10 was very. Having seen and eaten the dishes, they were asked again to indicate the best name for each using a second, anchored visual analogue scale. Biographical information including participant age and gender were recorded along with familiarity with eating in restaurants.

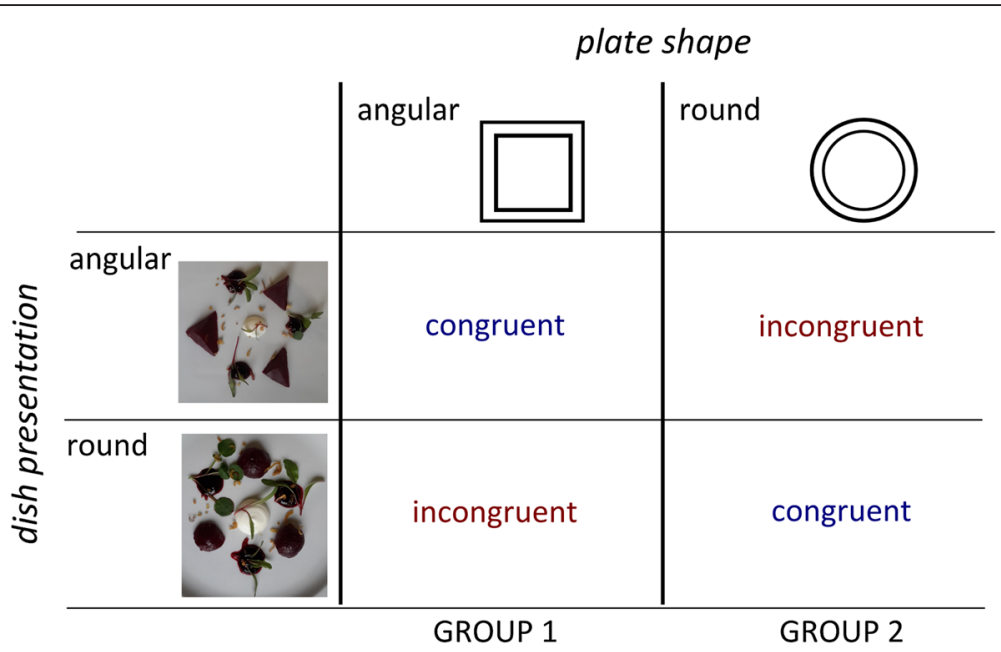

Figure 1 Study design showing manipulation of plate shape and serving presentation and resulting congruency of foreground (food presentation) and background (plate shape). 
Table 1 Behavioural results summary

\begin{tabular}{|c|c|c|c|c|c|c|c|c|}
\hline & & & \multicolumn{2}{|l|}{ Naming } & \multicolumn{4}{|l|}{ Flavour } \\
\hline & & & Sight & $\overline{\text { Sight/taste }}$ & Sweetness & Sourness & Intensity & Pleasantness \\
\hline \multicolumn{9}{|l|}{ Session 1} \\
\hline Congruent & Square plate & Angular presentation & $1.35 \pm 0.84$ & $3.425 \pm 1.22$ & $4.75 \pm 1.57$ & $4.31 \pm 2.09$ & $5.84 \pm 1.34$ & $7.13 \pm 1.75$ \\
\hline Incongruent & Square plate & Round presentation & $5.14 \pm 1.23$ & $4.43 \pm 1.51$ & $5 \pm 1.63$ & $4.19 \pm 1.64$ & $6 \pm 1.37$ & $7.16 \pm 2.11$ \\
\hline \multicolumn{9}{|l|}{ Session 2} \\
\hline Congruent & Round plate & Round presentation & $5.55 \pm 1.59$ & $4.53 \pm 1.93$ & $5.56 \pm 1.93$ & $5 \pm 2.24$ & $6.53 \pm 1.20$ & $7.44 \pm 1.50$ \\
\hline Incongruent & Round plate & Angular presentation & $1.55 \pm 1.64$ & $2.53 \pm 2.46$ & $5.31 \pm 1.96$ & $4.73 \pm 2.22$ & $6.38 \pm 1.09$ & $7.53 \pm 1.36$ \\
\hline
\end{tabular}

Mean subjective ratings across individuals by group and congruency condition for naming and flavour perception \pm standard deviation (SD).

\section{Results}

Within group, for both square and round plates, a repeated measures two-factor analysis of variance (ANOVA) showed a main effect of serving presentation on naming of the dish (square plates $\mathrm{F}(1,60)$ : $61.39, P<0.0001$; round plates $\mathrm{F}(1,60)$ : $36.86, P<0.0001)$. For both square plates and round plates, a significant interaction was observed for serving presentation and naming condition (by sight only or sight and taste) (square plates $\mathrm{F}(1,60): 20.81, P<0.0001$; round plates $F(1,60): 5.39, P<0.05$ ) (see Table 1 ). Figure 2 shows the nature of this interaction with ratings converging away from the extremes of the scale. We hypothesized that the observed change in rating across naming conditions (by sight only $v s$. after seeing and tasting) might be predicted by the nature of the flavour experience, as evidenced by ratings of intensity or pleasantness [4]. We see a
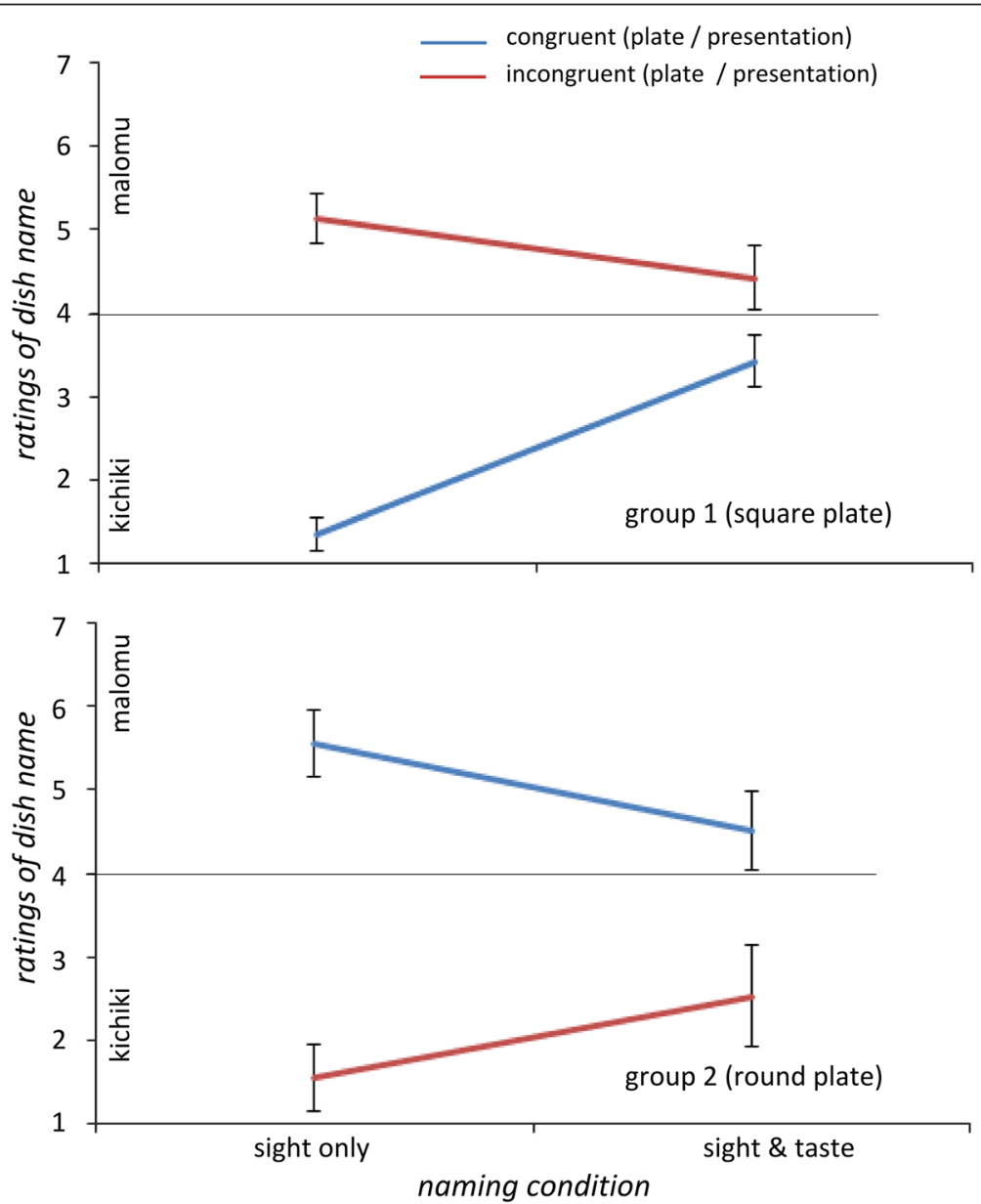

Figure 2 Effect of serving presentation and plate shape on naming of dish based on either sight alone or after seeing and tasting. Error bars represent standard error (SE). 


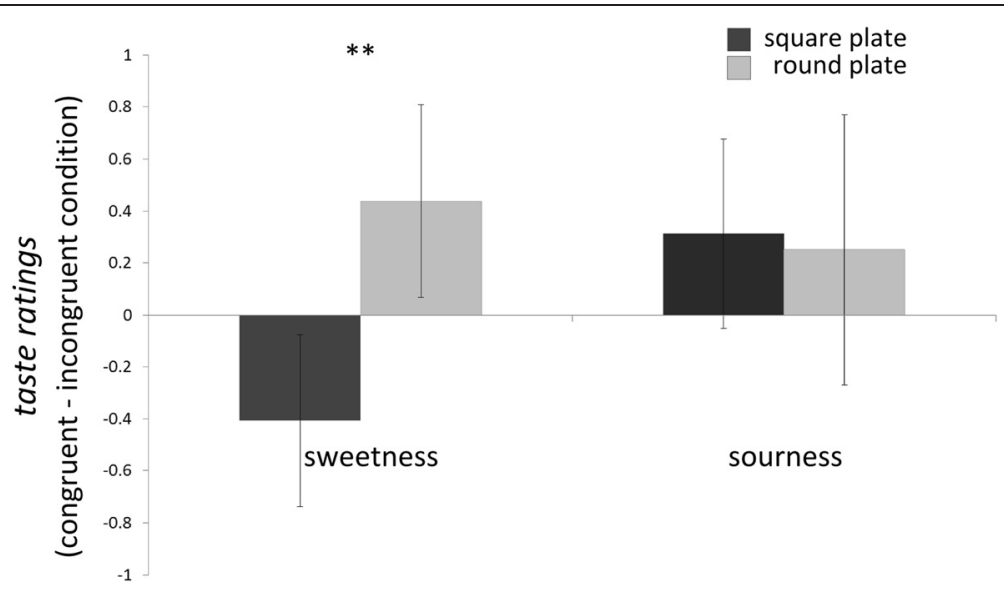

Figure 3 Effect of congruency of visual foreground (serving presentation) and background (plate shape) on changes in sweetness and sourness ratings (congruent - incongruent). ${ }^{* *}$ denotes two-tailed significance, $P<0.05$. Error bars represent standard error (SE).

significant correlation between pleasantness ratings and the change in naming ratings but only for congruent servings (square plates: $r=0.6, P=0.01$; round plates: $r=0.5, P$ $<0.05)$.

To test the effect of congruency on perceived taste, changes in subjective ratings (sweetness, sourness) were calculated between congruent and incongruent conditions (for example, con $_{\text {sweetness }}$ - incon sweetnesss $_{\text {s }}$. T-tests were performed identifying a significant difference in sweetness between square and round plate servings $(t(15)=-2.24$; $P<0.05$; Figure 3 ). No significant effect was observed for changes in sourness ratings.

\section{Discussion}

The present results shed new light on the effect of food presentation and the possibility to harness crossmodal correspondences to modulate people's overall experience of a complex dish. In particular, using the crossmodal correspondences between shapes and speech sounds, and shapes and flavours, this study allows us to disentangle the effects of plate shape and dish presentation on the diner's expectations and experience. The results confirmed that certain visual presentations of food were associated with unfamiliar names if they respected sound symbolism: Spherical beetroots displayed in a circle were preferentially called 'malomu' while angular beetroots displayed in a triangular way were preferentially called 'kichiki'. The results further show that the choice of the best name for a dish was driven by the presentation of the food itself and was little influenced by the shape of the plate. By contrast, the change in the perceived taste of the dishes was driven by the congruency between the shape of the plate and the shape and presentation of the dish: The same ingredients were rated as less sweet when their presentation was angular on an angular plate and sweeter in a rounded presentation on a round plate. The effect was significant for sweetness, but not for sourness, which might confirm the distinctive character of the roundness-sweetness correspondence: Velasco et al. [4] have shown that round shapes are exclusively matched to sweetness, while angular shapes can correspond to sourness, bitterness or saltiness. Liang et al. [19] have also found that round shapes have measurable perceptual effects and decrease the threshold for the detection of sweetness. In the absence of large scale cross-cultural studies [8], it is still difficult to determine how much of the associations between shapes and flavours or tastes are learned and whether they depend on people's preferences [4]. The universal character of sound symbolism, as well as the early effects of shape-smell congruence on sensory processing [20], still provides a good reason to believe that some of these effects can be due to general environmental characteristics and will exist across cultures.

Importantly, these results highlight the importance of food presentation on the experience of complex dishes [21], including the potential to make diners experience the same dish in different ways depending on the shape of the plate and the associations evoked by the food presentation. They also help drawing new guidelines regarding the choice of plate and names for new dishes.

\section{Abbreviation \\ ANOVA: analysis of variance.}

\section{Competing interests}

The authors declare that they have no competing interests.

\section{Authors' contributions}

MF, OD, DP and DO were involved in study design; MF, OD, DP and DO collected the data; MF analysed the data; and MF, OD, DP and DO were involved in writing the manuscript. All authors read and approved the final manuscript.

\section{Acknowledgements}

M. T. Fairhurst and O. Deroy are funded by the RTS-AHRC grant (AH-L007053/1) within the 'Science in Culture' scheme. The authors wish to thank the Being 
Human festival (2014) for providing the platform for the public event at which testing took place.

\section{Author details}

${ }^{1}$ Centre for the Study of the Senses, School of Advanced Study, University of London, London WC1E 7HU, UK. 'Experimental Kitchen, The Fat Duck, Bray SL6 2AQ, UK.

Received: 18 March 2015 Accepted: 14 April 2015

Published online: 15 May 2015

\section{References}

1. Simmons WK, Martin A, Barsalou LW. Pictures of appetizing foods activate gustatory cortices for taste and reward. Cereb Cortex. 2005;15:1602-8.

2. Deroy $\mathrm{O}$, Spence $\mathrm{C}$. Why we are not all synesthetes (not even weakly so). Psychon Bull Rev. 2013;20(4),643-64

3. Spence C, Ngo M. Assessing the shape symbolism of the taste, flavour, and texture of foods and beverages. Flavour. 2012;1:12.

4. Velasco C, Woods A, Deroy O, Spence C. Hedonic mediation of the crossmodal correspondence between taste and shape. Food Qual Prefer. 2015;41:151-8

5. Deroy $\mathrm{O}$, Valentin $\mathrm{D}$. Tasting liquid shapes: investigating cross-modal correspondences. Chemosens Percept. 2011;4:80-90.

6. Deroy O, Crisinel AS, Spence C. Crossmodal correspondences between odors and contingent features: odors, musical notes, and geometrical shapes. Psychon Bull Rev. 2013;20(5):878-96.

7. Ngo MK, Spence C. Assessing the shapes and speech sounds that people associate with different kinds of chocolate. J Sens Stud. 2011;26:421-8.

8. Bremner A, Caparos S, Davidoff J, de Fockert J, Linnell K, Spence C. Bouba and Kiki in Namibia? Western shape-symbolism does not extend to taste in a remote population. Cognition. 2013;126:165-72.

9. Ngo MK, Velasco C, Salgado A, Boehm E, O'Neill D, Spence C. Assessing crossmodal correspondences in exotic fruit juices: the case of shape and sound symbolism. Food Quality \& Preference. 2013;28:361-9.

10. Köhler W. Gestalt psychology. New York: Liveright; 1929.

11. Crisinel AS, Jones S, Spence C. "The sweet taste of maluma": crossmodal associations between tastes and words. Chemosens Percept. 2012;5:266-73.

12. Ramachandran VS, Hubbard EM. Synaesthesia - a window into perception, thought and language. J Conscious Stud. 2001;8:3-34.

13. Spence C. Managing sensory expectations concerning products and brands: capitalizing on the potential of sound and shape symbolism. J Consum Psychol. 2012;22:37-54.

14. Spence C, Gallace A. Tasting shapes and words. Food Quality \& Preference. 2011;22:290-5.

15. Stewart PC, Goss E. Plate shape and colour interact to influence taste and quality judgments. Flavour. 2013;2(1):27.

16. Piqueras-Fiszman B, Harrar V, Alcaide J, Spence C. Is it the plate or is it the food? Assessing the influence of the color (black or white) and shape of the plate on the perception of the food placed on it. Food Quality \& Preference. 2012;24:205-8.

17. Spence C, Piqueras-Fiszman B, Michel C, Deroy O. Plating manifesto (II): the art and science of plating. Flavour. 2014;3(1):4.

18. Nielsen A, Rendall D. The sound of round: evaluating the sound-symbolic role of consonants in the classic Takete-Maluma phenomenon. Can J Exp Psychol. 2011;65:115-24.

19. Liang P, Roy S, Chen ML, Zhang GH. Visual influence of shapes and semantic familiarity on human sweet sensitivity. Behav Brain Res. 2013;253:42-7.

20. Seo HS, Arshamian A, Schemmer K, Scheer I, Sander T, Ritter G, et al. Cross-modal integration between odors and abstract symbols. Neurosci Lett. 2010;478:175-8.

21. Deroy $\mathrm{O}$, Michel $\mathrm{C}$, Piqueras-Fiszman B, Spence $\mathrm{C}$. The plating manifesto (I): from decoration to creation. Flavour. 2014;3(1):1-11.

\section{Submit your next manuscript to BioMed Central and take full advantage of:}

- Convenient online submission

- Thorough peer review

- No space constraints or color figure charges

- Immediate publication on acceptance

- Inclusion in PubMed, CAS, Scopus and Google Scholar

- Research which is freely available for redistribution

Submit your manuscript at www.biomedcentral.com/submit 